\title{
A THEOREM ON GENERALIZED DERIVATIVES
}

\section{A. ZYGMUND}

1. General remarks. Suppose that a function $f(x)$, defined in the neighborhood of a point $x_{0}$, satisfies a relation

$$
\begin{aligned}
f\left(x_{0}+t\right)= & \alpha_{0}+\alpha_{1} t+\alpha_{2} t^{2} / 2 !+\cdots \\
& +\alpha_{k-1} t^{k-1} /(k-1) !+\omega_{k}(t) t^{k} / k !,
\end{aligned}
$$

where $\alpha_{0}, \alpha_{1}, \cdots, \alpha_{k-1}$ are independent of $t$, and the expression $\omega_{k}(t)=\omega\left(x_{0}, t\right)$ approaches a finite limit $\alpha_{k}$ as $t$ tends to 0 . The function $f$ is then said to possess a $k$ th generalized derivative at the point $x_{0}$, and $\alpha_{k}$ is the value of that derivative. Instead of $\alpha_{k}$ we shall write $D_{k} f\left(x_{0}\right)$. It is clear that the existence of $D_{k} f\left(x_{0}\right)$ implies that of $D_{k-1} f\left(x_{0}\right)$.

The existence of $D_{0} f\left(x_{0}\right)$ is simply continuity of the function $f$ at the point $x=x_{0}$. For $k=1$ the definition of $D_{k} f\left(x_{0}\right)$ is equivalent to that of the ordinary derivative $f^{(k)}\left(x_{0}\right)$. No such equivalence exists for higher values of $k$, for then the existence of $D_{k} f\left(x_{0}\right)$ does not even imply continuity of $f$ for $x \neq x_{0}$. However, if $f^{(k)}\left(x_{0}\right)$ exists and is finite, then $D_{k} f\left(x_{0}\right)$ also exists and is equal to $f^{(k)}\left(x_{0}\right)$.

It is a classical result of Fatou that, if a function $f(x)$ is everywhere continuous and, say, of period $2 \pi$, then the integral

$$
\int_{0}^{\pi} \frac{f(x+t)-f(x-t)}{t} d t=\lim _{\delta \rightarrow+0} \int_{\delta}^{\pi} \frac{f(x+t)-f(x-t)}{t} d t
$$

exists for almost every $x .{ }^{1}$ This integral may also be written

$$
\int_{0}^{\pi} \frac{\omega_{0}(x, t)-\omega_{0}(x,-t)}{t} d t
$$

or

$$
\int_{0}^{\pi} \frac{\epsilon_{0}(x, t)-\epsilon_{0}(x,-t)}{t} d t
$$

if for any $x$ for which $D_{k} f(x)$ exists we introduce the notation

$$
\omega_{k}(x, t)=D_{k} f(x)+\epsilon_{k}(x, t)
$$

(so that $\epsilon(x, t)$ tends to 0 with $t$, for $x$ fixed).

Received by the editors April 28, 1943.

${ }^{1}$ For this and more general results, see, for example, the author's Trigonometric Series, Warsaw, 1935, chap. 7. 
The convergence of the integral (1.3) may be due either to the smallness of the numerator $\epsilon_{0}(x, t)-\epsilon_{0}(x,-t)$ (which, anyway, tends to 0 ) or to the interference of positive and negative values of the integrand. In general, it is the second explanation which is right. For there exist continuous functions $f(x)$ such that the integral

$$
\int_{0}^{\pi} \frac{\left|\epsilon_{0}(x, t)-\epsilon_{0}(x,-t)\right|}{t} d t
$$

is infinite for every value of $x .^{2}$ For what follows it is of interest to observe that there exist continuous functions $f$ such that the integral

$$
\int_{0}^{\pi} \frac{\left|\epsilon_{0}(x, t)-\epsilon_{0}(x,-t)\right|^{r}}{t} d t
$$

is infinite for every $x$ and for every positive number $r$, however large. ${ }^{3}$ Since $\epsilon_{0}(x, t)-\epsilon_{0}(x,-t)$ tends to 0 with $t$, the divergence of (1.5) implies the divergence of the integral with any exponent smaller than $r$.

The result of Fatou has been generalized in several ways. In particular, Plessner showed that, if

(i) $f(x)$ is integrable over $(0,2 \pi)$ and is of period $2 \pi$;

(ii) $D_{k} f(x)$ exists for every point $x$ of a set $E$ of positive measure, then the integral

$$
\begin{aligned}
\int_{0}^{\pi} \frac{\omega_{k}(x, t)-\omega_{k}(x,-t)}{t} d t & =\int_{0}^{\pi} \frac{\epsilon_{k}(x, t)-\epsilon_{k}(x,-t)}{t} d t \\
& =\lim _{\delta \rightarrow+0} \int_{\delta}^{\pi}
\end{aligned}
$$

exists for almost every $x \in E .^{4}$

The most interesting is, of course, the behavior of the integrand $\left[\epsilon_{k}(x, t)-\epsilon_{k}(x,-t)\right] / t$ in the neighborhood of $t=0$. Since the existence of $D_{k} f\left(x_{0}\right)$ implies the boundedness of $f$ in the neighborhood of the point $x_{0}$, the assumption of integrability of $f$ in Plessner's theorem is really no restriction of generality, and is made merely to simplify the statement.

${ }^{2}$ See, for example, S. Kaczmarz, Integrale vom Dinischen Typus, Studia Mathematica vol. 3 (1931) pp. 189-199, or the author's Trigonometric Series, p. 77.

Kaczmarz shows that the set of continuous functions $f$ for which the integral (1.4) is finite for some $x$ is of the first category in the space of all continuous functions.

${ }^{3}$ The proof does not differ from that of the special case $r=1$.

4 Plessner, Ueber das Verhalten anylytischer Funktionen auf dem Rande des Definitionsbereiches, J. Reine Angew. Math. vol. 158 (1927) pp. 219-227. A different proof will be found in Marcinkiewicz, Sur le sêries de Fourier, Fund. Math. vol. 27 (1936) pp. 38-69. 
Recently, Marcinkiewicz proved the following remarkable result. Suppose that a function $f(x)$, of integrable square and of period $2 \pi$, has a finite derivative for every point $x$ of a set $E$ of positive measure. Then the integral

$$
\int_{0}^{\pi} \frac{[f(x+t)+f(x-t)-2 f(x)]^{2}}{t^{3}} d t
$$

is finite for almost every $x \in E .^{5}$

Consideration of this integral was suggested by its analogy with an important function

$$
g(\theta)=\left\{\int_{0}^{1}(1-\rho)\left|F^{\prime}\left(\rho e^{i \theta}\right)\right|^{2} d \rho\right\}^{1 / 2} \quad(F(z) \text { regular in }|z|<1)
$$

introduced by Littlewood and Paley. ${ }^{6}$ However, the integral (1.7) may also be given a different interpretation. For, if we note that

$$
\begin{aligned}
f(x+t)+f(x-t)-2 f(x) & =t\left[\omega_{1}(x, t)-\omega_{1}(x,-t)\right] \\
& =t\left[\epsilon_{1}(x, t)-\epsilon_{1}(x,-t)\right]
\end{aligned}
$$

at every point where $f^{\prime}(x)$ exists, we may write the integral (1.7) in the form

$$
\int_{0}^{\pi} \frac{\left[\omega_{1}(x, t)-\omega_{1}(x,-t)\right]^{2}}{t} d t=\int_{0}^{\pi} \frac{\left[\epsilon_{1}(x, t)-\epsilon_{1}(x,-t)\right]^{2}}{t} d t
$$

The finiteness of this integral at almost every point where $f^{\prime}$ exists, as compared with the fact that there exist continuous functions $f$ such that

$$
\int_{0}^{\pi} \frac{\left[\epsilon_{0}(x, t)-\epsilon_{0}(x,-t)\right]^{2}}{t} d t=+\infty \text { for every } x
$$

(cf. (1.5)), indicates that the behavior of the remainders $\epsilon_{0}(x, t)$ and $\epsilon_{1}(x, t)$ is essentially different. It also raises the problem of the extension of Marcinkiewicz's result to functions with a $k$ th generalized derivative. The answer to that problem is given by the following theorem, the proof of which is the main object of this note, and which reduces to Marcinkiewicz's theorem for $k=1$.

THEOREM. Suppose that a function $f(x)$ is of the class $L^{2}$ and of period $2 \pi$, and that the generalized derivative $D_{k} f(x)$ exists for every point $x$ of

5 Marcinkiewicz, Sur quelques intégrales du type de Dini, Annales de la Société Polonaise de Mathématiques, 1938, pp. 42-50.

${ }^{6} \mathrm{~J}$. E. Littlewood and R. E. A. C. Paley, Theorems on Fourier series and power series, Proc. London Math. Soc. vol. 42 (1937) pp. 52-89. 
$a$ set $E$ of positive measure $(k=1,2, \cdots)$. Let $\omega_{k}(x, t)$ and $\epsilon_{k}(x, t)$ be defined by the equations

$$
\begin{aligned}
f(x+t) & =\sum_{\nu=0}^{k-1} D_{v} f(x) t^{\nu} / \nu !+\omega_{k}(x, t) t^{k} / k !, \\
\omega_{k}(x, t) & =D_{k} f(x)+\epsilon_{k}(x, t),
\end{aligned}
$$

$x \in E$.

Then the integral

$$
\int_{0}^{\pi} \frac{\left[\omega_{k}(x, t)-\omega_{k}(x,-t)\right]^{2}}{t} d t=\int_{0}^{\pi} \frac{\left[\epsilon_{k}(x, t)-\epsilon_{k}(x,-t)\right]^{2}}{t} d t
$$

is finite for almost every $x \in E$.

This result complements the theorem of Plessner stated above, but at individual points is not comparable with the latter. It displays a new property of the remainder $\omega_{k}(x, t)$, a property which, unlike that in Plessner's theorem, is expressed by the convergence of a positive integral.

2. Auxiliary lemmas. The proof of the theorem depends on two known lemmas. ${ }^{7}$

Lemma 1. Suppose that $P$ is a perfect set of positive measure situated in the interval $(0,2 \pi)$ and continued periodically. Let $\phi(x)=\phi_{P}(x)$ denote the function which is equal to 0 over $P$, and is equal to $d$ if $x$ belongs to an interval continguous to $P$ and of length $d$. Then, whatever $\lambda>0$, the integral $\int_{-\pi}^{\pi}\left(\phi^{\lambda}(x+t) /|t|^{\lambda+1}\right) d t$ is finite for almost every $x \in P$.

Lemma 2. Suppose that a function $f(x)$ of period $2 \pi$ has a kth generalized derivative for every point $x$ of a set $E$ of positive measure. Then $f(x)=g(x)+h(x)$, where

(i) $g(x)$ has everywhere a continuous kth derivative $g^{(k)}(x)$;

(ii) $g(x)=f(x)$ in a perfect set $P$ contained in $E$ and of positive measure;

(iii) except, perhaps, for a few segments $\Delta$ contiguous to $P$,

$$
\underset{x \in \Delta}{M \operatorname{ax}_{\Delta}}|h(x)| \leqq M \Delta^{k},
$$

with $M$ independent of $\Delta .^{9}$

${ }^{7}$ Both lemmas were used by Marcinkiewicz in his proof of the finiteness of the integral (1.7), so that the proof given here of the theorem is partly modelled on Marcinkiewicz's argument.

${ }^{8} \mathrm{~J}$. Marcinkiewicz, the paper cited in footnote 4.

$9 \mathrm{~J}$. Marcinkiewicz, loc. cit. Marcinkiewicz's argument shows that the set $P$ may be of measure arbitrarily close to that of $E$. 
Passing to the proof of the theorem, let us assume first that the function $f(x)$ has a continuous $k$ th derivative $f^{(k)}(x)$. To fix the ideas we assume that $k$ is even. Then

$$
\begin{aligned}
(1 / 2)[f(x+t)-f(x-t)]= & f^{\prime}(x) t+f^{\prime \prime \prime}(x) t^{3} / 3 !+\cdots \\
& +f^{(k-1)}(x) t^{k-1} /(k-1) ! \\
& +(1 / 2)\left[\omega_{k}(x, t)-\omega_{k}(x,-t)\right] t^{k} / k ! .
\end{aligned}
$$

Let us now consider the Fourier series of the function $f(x)$. It may be written in the form

$$
f(x) \sim \sum_{\nu=-\infty}^{+\infty} c_{\nu}(i v)^{-k} e^{i \nu x},
$$

if we assume, for simplicity, that the constant term of the Fourier series is zero. Of course,

$$
\sum_{\nu=-\infty}^{+\infty}\left|c_{\nu}\right|^{2}<+\infty
$$

and the Fourier series of $f^{(l)}(x), 0 \leqq l \leqq k$, is obtained by differentiating $l$ times the Fourier series of $f(x)$. Hence, taking into account that for fixed $t,(1 / 2)[f(x+t)-f(x-t)] \sim \sum_{\nu=-\infty}^{+\infty} i c_{\nu}(i \nu)^{-k} e^{i v x} \sin \nu t$, we get (cf. (2.1))

$$
\begin{aligned}
& \frac{1}{2 \cdot k !}\left[\omega_{k}(x, t)-\omega_{k}(x,-t)\right] \\
& \sim \sum_{\nu=-\infty}^{+\infty} c_{\nu} e^{i \nu x} \frac{1}{(i \nu t)^{k}}\left\{i \sin \nu t-\left(i \nu t+\frac{(i \nu t)^{3}}{3 !}+\cdots+\frac{(i \nu t)^{k-1}}{(k-1) !}\right)\right\} \\
&(2.2)=i(-1)^{k / 2} \sum_{\nu=-\infty}^{+\infty} c_{\nu} e^{i \nu x} \frac{1}{(\nu t)^{k}} \cdot\left\{\sin \nu t-\left[\nu t-\frac{(\nu t)^{3}}{3 !}+\cdots \pm \frac{(\nu t)^{k-1}}{(k-1) !}\right]\right\} \\
&=i(-1)^{k / 2} \sum_{\nu=-\infty}^{+\infty} c_{\nu} e^{i \nu x} \xi_{k}(\nu t),
\end{aligned}
$$

say, where

$$
\xi_{k}(u)=\left[\sin u-\left(u-\frac{u^{3}}{3 !}+\cdots \pm \frac{u^{k-1}}{(k-1) !}\right)\right] / u^{k} .
$$

Let us divide (2.2) by $t^{1 / 2}$ and apply Parseval's formula to the resulting relation. We get 


$$
\frac{1}{8 \pi(k !)^{2}} \int_{-\pi}^{\pi} \frac{\left[\omega_{k}(x, t)-\omega_{k}(x,-t)\right]^{2}}{t} d x=\sum_{\nu=-\infty}^{+\infty}\left|c_{\nu}\right|^{2} \frac{\xi_{k}^{2}(\nu t)}{t} .
$$

Integrating both sides with respect to $t$ over the interval $(0, \pi)$, and inverting the order of integration on the left, we see that

$$
\begin{aligned}
& \frac{1}{8 \pi(k !)^{2}} \int_{-\pi}^{\pi} d x\left\{\int_{0}^{\pi} \frac{\left[\omega_{k}(x, t)-\omega_{k}(x,-t)\right]^{2}}{t} d t\right\} \\
& \quad=\sum_{\nu=-\infty}^{+\infty}\left|c_{\nu}\right|^{2} \int_{0}^{\pi} \frac{\xi_{k}^{2}(\nu t)}{t} d t=\sum_{\nu=-\infty}^{+\infty}\left|c_{\nu}\right|^{2} \int_{0}^{\nu \pi} \frac{\xi_{k}^{2}(u)}{u} d u .
\end{aligned}
$$

Let us now observe that the integral.

$$
\int_{0}^{\infty} \frac{\xi_{k}^{2}(u)}{u} d u
$$

is finite. This follows from the fact that in the neighborhood of $u=0$ the integrand is $\left(u^{-1}\right) \cdot O\left(u^{2}\right)=O(u)=O(1)$, and in the neighborhood of $u=+\infty$ it is $u^{-1} \cdot O\left(u^{2 k-2}\right) u^{-2 k}=O\left(u^{-3}\right)$.

Since $\sum\left|c_{\nu}\right|^{2}<+\infty$, the right-hand side of (2.3) is finite, so that the integral

$$
\int_{0}^{\pi} \frac{\left[\omega_{k}(x, t)-\omega_{k}(x,-t)\right]^{2}}{t} d t
$$

is finite for almost every $x$.

If $k$ is odd, the argument is similar. Instead of (2.1) we consider the formula

$$
\begin{aligned}
(1 / 2)[f(x+t)+f(x-t)]= & f(x)+f^{\prime \prime}(x) t^{2} / 2 !+\cdots \\
& +f^{(k-1)}(x) t^{k-1} /(k-1) ! \\
& +(1 / 2)\left[\omega_{k}(x, t)-\omega_{k}(x,-t)\right] t^{k} / k !
\end{aligned}
$$

and we still get (2.3), where now

$$
\xi_{k}(u)=\left[\cos u-\left(1-u^{2} / 2 !+\cdots \pm u^{k-1} /(k-1) !\right)\right] u^{-k}
$$

and the integral (2.4) is again finite. Thus the theorem is proved if $f^{(k)}(x)$ exists everywhere and is continuous.

3. Proof of the theorem. In order to complete the proof of the theorem, let us assume that there is a periodic function $f \in L^{2}$ having a generalized derivative $D_{k} f(x)$ at every point of a set $E$ of positive measure, and such that the integral (1.9) diverges everywhere in $E$. Let us now consider the decomposition $f=g+h$ of Lemma 2. The function $g$ having everywhere a continuous derivative $g^{(k)}(x)$, the cor- 
responding integral (1.9) is convergent almost everywhere, and so almost everywhere in $E$. If we could prove that the integral (1.9) corresponding to the function $h$ is finite in a set $E^{\prime}$ contained in $E$ and of positive measure, it would follow that the integral (1.9) for the function $f$ is convergent almost everywhere in $E^{\prime}$, contrary to the assumption that it is divergent at every point of $E$. That contradiction would prove the theorem.

Let us therefore consider the function $h$ of Lemma 2. That function vanishes over a perfect set $P \subset E$. Part (iii) of Lemma 2 asserts that for all, except perhaps a finite number, of the intervals $\Delta$ contiguous to $P$,

$$
|h(x)| \leqq M \phi^{k}(x), \quad x \in \Delta .
$$

This inequality is also valid for the points of the set $P$, since both $h(x)$ and $\phi(x)$ vanish over $P$.

Let $x_{0}$ be any point of density of $P$ (hence $x_{0}$ is not an end point of any interval contiguous to $P$ ). From the definition of the function $\phi(x)$ it follows that $\phi\left(x_{0}+t\right)=o(|t|)$ as $t \rightarrow 0$. Since (3.1) is valid in a neighborhood $\left(x_{0}-\delta, x_{0}+\delta\right)$ of $x_{0}$, we see that $h\left(x_{0}+t\right)=o\left(|t|^{k}\right)$ as $t \rightarrow 0$. Hence the function $\omega_{k}\left(x_{0}, t\right)$ corresponding to $h(x)$ is simply $k ! h\left(x_{0}+t\right) t^{-k}$. Thus, in our case,

$$
\begin{aligned}
\int_{0}^{\delta} \frac{\left[\omega_{k}\left(x_{0}, t\right)-\omega_{k}\left(x_{0},-t\right)\right]^{2}}{t} d t & \\
& \leqq 2 \int_{0}^{\delta} \frac{\omega_{k}^{2}\left(x_{0}, t\right)}{t} d t+2 \int_{0}^{\delta} \frac{\omega_{k}^{2}\left(x_{0},-t\right)}{t} d t \\
& =2(k !)^{2} \int_{-\delta}^{+\delta} \frac{h^{2}\left(x_{0}+t\right)}{|t|^{2 k+1}} d t \\
& \leqq 2 M^{2}(k !)^{2} \int_{-\delta}^{+\delta} \frac{\phi^{2 k}\left(x_{0}+t\right)}{|t|^{2 k+1}} d t
\end{aligned}
$$

If, therefore, $E^{\prime}$ is the set of points of density of $P$ for which the integral

$$
\int_{-\pi}^{\pi} \frac{\phi^{2 k}(x+t)}{|t|^{2 k+1}} d t
$$

is finite, the integral (1.9) corresponding to the function $h$ converges at every point of $E^{\prime}$. This completes the proof of the theorem, since the set $E^{\prime}$ is obviously of positive measure.

MT. Holyoke College 